\title{
CULTURA DE SEGURANÇA DO PACIENTE EM CENTRO CIRÚRGICO: PERCEPÇÃO DA EQUIPE DE ENFERMAGEM
}

\author{
Patient safety culture in surgicenters: perception of nursing team
}

La cultura de seguridad del paciente en centro quirúrgico: la percepción del equipo de enfermería

Elena Bohomol ${ }^{1 *} \mathbb{D}$, Eliana Ferreira de Melo² (D)

RESUMO: Objetivo: Analisar a percepção de profissionais de enfermagem de um centro cirúrgico em um hospital privado acerca das dimensões da cultura de segurança do paciente. Método: Estudo descritivo e exploratório, com abordagem quantitativa, realizado em uma instituição hospitalar privada e acreditada, com 37 profissionais de enfermagem do centro cirúrgico, utilizando o instrumento Hospital Survey on Patient Safety Culture para coleta de dados. Resultados: Identificou-se a dimensão "Aprendizado organizacional e melhoria contínua" (77,4\%) como área forte na instituição. Encontraram-se quatro áreas frágeis, referentes às dimensões: "Trabalho em equipe dentro das unidades" (47,4\%), "Abertura da comunicação" (45,8\%), "Resposta não punitiva aos erros" (29,2\%) e "Adequação de pessoal" (42\%). Conclusão: Há a necessidade de se implementar mudanças que requeiram esforços de toda a organização hospitalar nos níveis estratégico, administrativo e operacional, principalmente para incentivar a atenção dos profissionais na condução das ações que fortaleçam a cultura não punitiva, e estudar o dimensionamento de profissionais para o atendimento do paciente no perioperatório. Palavras-chave: Enfermagem perioperatória. Segurança do paciente. Cultura organizacional.

ABSTRACT: Objective: To analyze the perception of nursing professionals from a surgicenter at a private hospital of patient's safety culture dimensions. Method: This is a descriptive and exploratory study with a quantitative approach, conducted in a private and accredited hospital institution, including 37 nursing professionals from surgicenters. It used the Hospital Survey on Patient Safety Culture instrument for data collection. Results: The dimension "Organizational learning and continuous improvement" was identified as a strong area (77.4\%) in the institution. We found four weak areas regarding the dimensions: "Teamwork within units" (47.4\%); "Communication opening" (45.8\%); "Nonpunitive response to errors" (29.2\%); and "Team Adequacy" (42\%). Conclusion: Implementing changes that require efforts from the entire hospital institution at the strategic, administrative, and operational levels are required, mainly to encourage professionals' attention when conducting actions that strengthen a nonpunitive culture, and to study their dimensioning regarding patient's care during the perioperative period.

Keywords: Perioperative nursing. Patient safety. Organizational culture.

RESUMEN: Objetivo: Analizar la percepción de los profesionales de enfermería de un centro quirúrgico en un hospital privado sobre las dimensiones de la cultura de seguridad del paciente. Método: Estudio descriptivo y exploratorio con enfoque cuantitativo, realizado en una institución hospitalaria privada y acreditada, incluyendo 37 profesionales de enfermería del centro quirúrgico, utilizándose el instrumento Hospital Survey on Patient Safety Culture para la recolección de datos. Resultados: La dimensión "Aprendizaje organizacional y mejora continua” se identificó como un área fuerte (77,4\%) en la institución. Se encontraron cuatro áreas frágiles con respecto a las dimensiones: “Trabajo en equipo dentro de las unidades” (47,4\%); “Comunicación abierta” (45,8\%); "Respuesta no punitiva a errores" (29,2\%) y "Adecuación del equipo" (42\%). Conclusión: Es necesario implementar cambios que requieran esfuerzos de toda la organización hospitalaria a los niveles estratégico, administrativo y operativo, especialmente para alentar la atención de los profesionales en la realización de acciones que fortalezcan la cultura no punitiva y para estudiar el dimensionamiento de los profesionales a la atención al paciente en el perioperatorio. Palabras clave: Enfermería perioperatoria. Seguridad del paciente. Cultura organizacional.

'Enfermeira. Professora associada livre-docente da Universidade Federal de São Paulo (Unifesp) - São Paulo (SP), Brasil.

'Enfermeira. Coordenadora de Enfermagem do Hospital São Camilo - São Paulo (SP), Brasil.

*Autor correspondente: ebohomol@unifesp.br

Recebido: 23/10/2018 - Aprovado: 10/06/2019

DOI: $10.5327 / 21414-4425201900030004$ 


\section{INTRODUÇÃo}

A segurança do paciente é definida pela Organização Mundial da Saúde (OMS) como "a redução do risco de danos desnecessários associados à assistência em saúde até um mínimo aceitável”. Compreende-se como mínimo aceitável aquilo que é viável diante do conhecimento atual, dos recursos disponíveis e do contexto em que a assistência foi realizada ${ }^{1}$.

Os erros e eventos adversos ocorrem em todos os cenários de assistência, incluindo os centros cirúrgicos (CC), que são considerados setores complexos e de alto risco ${ }^{2}$. Os processos de trabalho nesse setor envolvem uma gama de profissionais, práticas complexas, condições ambientais específicas, disponibilidade de recursos materiais e tecnológicos e prontidão no atendimento para a assistência segura, no entanto incidentes ocorrem e podem trazer danos aos pacientes, além de sofrimento e estresse aos profissionais que atuam nesse contexto ${ }^{3}$.

Organizações de saúde têm implantado iniciativas voltadas aos protocolos de cirurgia segura para garantir que os padrões estabelecidos sejam observados durante os procedimentos, que os eventos adversos apresentados na sala de cirurgia e na recuperação pós-anestésica sejam registrados de forma efetiva, que haja adequada atenção ao paciente no transoperatório e que a comunicação entre os profissionais seja efetiva ${ }^{4,5}$. Todavia, essas ações devem estar fundamentadas no amadurecimento gerencial, no envolvimento das lideranças, na integração de equipes e estratégias para eliminar a cultura da punição ${ }^{6}$.

Estudos demonstram que o ambiente do CC é um cenário com inúmeros conflitos e apontam para a frequência de comportamentos inadequados e arrogantes entre equipes ou condições inadequadas de trabalho, os quais podem afetar, negativamente, ou comprometer, potencialmente, o atendimento ao paciente ${ }^{5,6}$.

Nesse sentido, conhecer a cultura de segurança do paciente nesse cenário é aspecto imprescindível para efetivar melhorias $^{2,3}$. A cultura de segurança representa o conjunto de valores, atitudes, competências e comportamentos que determinam o comprometimento com a gestão da saúde e da segurança, substituindo a culpa e a punição pela oportunidade de aprender com as falhas e melhorar a atenção à saúde ${ }^{7}$.

Quanto maior a compreensão da equipe assistencial sobre os valores e as normas que regem a instituição e quanto mais os processos e sistemas estiverem adequados, mais seguro será o cuidado ${ }^{8}$. Reforçando esse entendimento, uma das premissas para a implantação do Programa Nacional de
Segurança do Paciente (PNSP) trata da importância de promover a cultura de segurança com foco no aprimoramento organizacional, no envolvimento dos profissionais e pacientes, na promoção de sistemas seguros e em mudanças nos processos de responsabilização individual ${ }^{9}$.

A equipe de enfermagem está completamente envolvida na assistência perioperatória, participa da atenção à equipe cirúrgica e tem a responsabilidade de promover um ambiente com qualidade e segurança. Portanto, é importante que se conheça a percepção da cultura de segurança entre profissionais de enfermagem que atuam em CC.

\section{OBJETIVO}

Analisar a percepção de profissionais de enfermagem de um CC em um hospital privado acerca das dimensões da cultura de segurança do paciente.

\section{MÉTODO}

Estudo descritivo e exploratório, com abordagem quantitativa, realizado em uma instituição hospitalar privada sem fins lucrativos, acreditada por programas de acreditação nacional (Organização Nacional de Acreditação) e internacionais (Health Standards Organization e Joint Commission International). O CC contava com estrutura para o atendimento de procedimentos de alta complexidade, incluindo cirurgia bariátrica, ortopédica, oncológica e cardíaca, além de procedimentos minimamente invasivos e de alta precisão, como videolaparoscopia, neuronavegação e demais técnicas videoassistidas.

Todos os profissionais de enfermagem do CC foram convidados a participar da pesquisa. Foram incluídos os que realizavam atividades assistenciais com contato direto com o paciente e excluídos aqueles que estavam de férias, folgas ou licenças e os enfermeiros que possuíam cargo de chefia. A população do estudo foi composta de 51 colaboradores, distribuídos em oito enfermeiros, 39 técnicos de enfermagem e quatro auxiliares de enfermagem.

O instrumento para coleta de dados foi o Hospital Survey on Patient Safety Culture (HSOPSC), da Agency for Healthcare Research \& Quality (AHRQ), entidade americana voltada para o desenvolvimento de estudos na área, principalmente em relação à implantação da cultura de segurança nas instituições de saúde. Trata-se de um instrumento mundialmente 
utilizado, tanto em hospitais quanto em outros tipos de instituições de saúde, idealizado pelas pesquisadoras Sorra e Nieva ${ }^{10} \mathrm{e}$ validado para a realidade brasileira por Reis et al. ${ }^{11}$. Contém nove seções, totalizando 42 itens relacionados à cultura de segurança do paciente e que medem 12 dimensões, divididas em três níveis:

- Nível Hospitalar:

1. Apoio da gestão hospitalar para segurança do paciente;

2. Trabalho em equipe entre as unidades;

3. Passagem de plantão/turno e transferências internas;

- Nível Unidade:

4. Expectativas do supervisor/chefe e ações promotoras de segurança;

5. Aprendizado organizacional e melhoria contínua;

6. Trabalho em equipe dentro das unidades;

7. Abertura da comunicação;

8. Retorno da informação e comunicação sobre os erros;

9. Resposta não punitiva aos erros;

10. Adequação de pessoal;

- Nível Resultados:

11. Percepção geral de segurança do paciente;

12. Frequência de eventos relatados.

O instrumento para a coleta de dados constituiu-se de duas partes: a primeira com itens relacionados a informações sociodemográficas (categoria profissional, escolaridade, turno de trabalho, tempo de trabalho na instituição e no setor e tempo de formação), e a segunda com itens que abrangem as 12 dimensões de cultura de segurança.

O instrumento foi disponibilizado de forma online, utilizando o software Interact ${ }^{\mathbb{R}}$, no período de 12 a 24 de julho de 2015, quando foi entregue o Termo de Consentimento Livre e Esclarecido (TCLE) e foram explicitados os propósitos do estudo.

A análise dos dados foi realizada por meio de estatística descritiva para as variáveis categóricas, apresentadas por frequências absolutas e relativas. As comparações das médias dos domínios e as variáveis de caracterização foram realizadas por meio de análise de variância (ANOVA), para comparação de três ou mais fatores. As análises foram interpretadas considerando nível de significância de $5 \%(\mathrm{p}=0,05)$ e realizadas com auxílio do software R 3.2.3.
Para o cálculo dos escores das dimensões, utilizaram-se os valores de 1 a 5 pontos para cada nível de resposta na ordem apresentada: discordo totalmente, discordo, não concordo nem discordo, concordo e concordo totalmente, e também para as respostas nunca, raramente, às vezes, quase sempre e sempre. Como o questionário possui a técnica de escala de Likert, os itens redigidos de forma negativa foram invertidos para a análise e pontuados na ordem de 5 a 1 . Para a composição do percentual de respostas positivas das 12 dimensões, foi utilizada a fórmula recomendada pela AHRQ, em que se calcula o número de respostas positivas dos itens da dimensão dividido pelo número total de respostas válidas (positivas, neutras e negativas). As respostas positivas representam uma reação assertiva no que tange à cultura de segurança do paciente. Classificam-se em: áreas fortes (escores superiores a 75\%), neutras (entre 50 e $75 \%$ ) e frágeis (escores inferiores a 50\%) da cultura de segurança. Os dados foram descritos e analisados por dimensão ${ }^{10}$.

A consistência interna das dimensões do HSOPSC foi avaliada pelo coeficiente alfa de Cronbach.

Este estudo seguiu os preceitos da Resolução no 466/2012, do Conselho Nacional de Saúde, e foi aprovado pelo Comitê de Ética em Pesquisa da Universidade Federal de São Paulo e da instituição participante, conforme parecer $n^{\circ} 655.946$.

\section{RESULTADOS}

Participaram do estudo 37 profissionais, correspondendo a $72,5 \%$ da população, sendo sete $(18,9 \%)$ enfermeiros e 30 $(81,1 \%)$ técnicos de enfermagem. Verificou-se que $65,9 \%$ deles possuem ensino médio; $48,6 \%$ trabalham no período da tarde; $48,6 \%$ possuem entre 1 e 5 anos na instituição; $54,1 \%$ trabalham entre 1 e 5 anos no setor e $35,1 \%$ são formados entre 6 e 10 anos (Tabela 1).

O coeficiente alfa de Cronbach do HSOPSC variou de 0,45 a 0,91 , e para o instrumento como um todo o referido coeficiente foi de 0,64 , o que confere ao questionário confiabilidade satisfatória.

A Tabela 2 apresenta a frequência das respostas positivas, neutras e negativas referentes ao nível e às dimensões de cultura de segurança do paciente. Em relação às respostas positivas, verificou-se que a dimensão "Aprendizado organizacional e melhoria contínua” (77,4\%) é a área forte na instituição.

As dimensões "Apoio da gestão hospitalar para segurança do paciente" (70,5\%), "Trabalho em equipe entre as unidades" (50,8\%) e "Passagem de plantão/turno e transferência" (50,7\%), do nível Hospitalar, encontram-se na área neutra no tocante à segurança do paciente. Também são áreas neutras as dimensões 
Tabela 1. Variáveis sociodemográficas dos profissionais do centro cirúrgico.

\begin{tabular}{|c|c|c|c|}
\hline \multicolumn{2}{|l|}{ Variáveis } & $\mathbf{N}$ & $\%$ \\
\hline \multirow{2}{*}{ Cargo/função } & Enfermeiro assistencial & 7 & 18,9 \\
\hline & Técnico em Enfermagem & 30 & 81,1 \\
\hline \multirow{3}{*}{ Escolaridade } & Ensino médio & 24 & 64,9 \\
\hline & Ensino superior & 8 & 21,6 \\
\hline & Especialização & 5 & 13,5 \\
\hline \multirow{3}{*}{ Período } & Manhã & 15 & 40,5 \\
\hline & Tarde & 18 & 48,6 \\
\hline & Noite & 4 & 10,8 \\
\hline \multirow{5}{*}{$\begin{array}{l}\text { Tempo na } \\
\text { instituição }\end{array}$} & Menos de 1 ano & 2 & 5,4 \\
\hline & 1 a 5 anos & 18 & 48,6 \\
\hline & 6 a 10 anos & 12 & 32,4 \\
\hline & 11 a 15 anos & 2 & 5,4 \\
\hline & 16 a 20 anos & 3 & 8,1 \\
\hline \multirow{5}{*}{$\begin{array}{l}\text { Tempo no } \\
\text { setor }\end{array}$} & Menos de 1 ano & 2 & 5,4 \\
\hline & 1 a 5 anos & 20 & 54,1 \\
\hline & 6 a 10 anos & 10 & 27 \\
\hline & 11 a 15 anos & 2 & 5,4 \\
\hline & 16 a 20 anos & 3 & 8,1 \\
\hline \multirow{5}{*}{$\begin{array}{l}\text { Tempo de } \\
\text { formação }\end{array}$} & 1 a 5 anos & 9 & 24,3 \\
\hline & 6 a 10 anos & 13 & 35,1 \\
\hline & 11 a 15 anos & 7 & 18,9 \\
\hline & 16 a 20 & 5 & 13,5 \\
\hline & 21 anos ou mais & 3 & 8,1 \\
\hline
\end{tabular}

"Expectativas do supervisor/chefe e ações promotoras de segurança" (56,1\%) e "Retorno da informação e comunicação sobre os erros" (50,1\%), do nível Unidade, e "Percepção geral de segurança do paciente" (60,0\%) e "Frequência de eventos relatados" (73,2\%), do nível Resultados.

Encontraram-se como áreas frágeis quatro dimensões do nível Unidade: "Trabalho em equipe dentro das unidades" (47,4\%), "Abertura da comunicação" (45,8\%), "Resposta não punitiva aos erros" (29,2\%) e "Adequação de pessoal” (42,0\%).

\section{DISCUSSÃO}

Os achados do estudo permitiram conhecer as características dos profissionais de enfermagem que atuam em um CC e os aspectos relevantes da cultura da segurança do paciente que precisam ser aprimorados.

Em relação à caracterização dos profissionais, destacou-se o expressivo número de técnicos em Enfermagem que trabalham no setor, alguns com formação em ensino superior. Esse aspecto corroborou os achados da pesquisa sobre o perfil da enfermagem brasileira, que demonstrou que os trabalhadores de nível médio apresentavam escolaridade acima da exigida para o desempenho de suas funções, apresentando o percentual de $11,7 \%$ de formados em cursos de graduação ${ }^{12}$.

Verificou-se que a maioria dos profissionais tinha, no máximo, 5 anos de atuação na instituição, dado semelhante ao encontrado em estudos nacionais e estrangeiros com a equipe de enfermagem de um $\mathrm{CC}^{8,13}$.

Tabela 2. Respostas positivas, neutras e negativas referentes ao nível e às dimensões de cultura de segurança do paciente.

\begin{tabular}{|c|c|c|c|c|}
\hline Nível & Dimensão & $\begin{array}{c}\text { Negativo } \\
\%\end{array}$ & $\begin{array}{c}\text { Neutro } \\
\%\end{array}$ & $\begin{array}{c}\text { Positivo } \\
\%\end{array}$ \\
\hline \multirow{3}{*}{ Hospitalar } & Apoio da gestão hospitalar para segurança do paciente & 10,5 & 19,0 & 70,5 \\
\hline & Trabalho em equipe entre as unidades & 24,4 & 24,8 & 50,8 \\
\hline & Passagem de plantão/turno e transferência & 25,3 & 24,0 & 50,7 \\
\hline \multirow{7}{*}{ Unidade } & Expectativas do supervisor/chefe e ações promotoras de segurança & 23,0 & 20,9 & 56,1 \\
\hline & Aprendizado organizacional e melhoria contínua & 9,9 & 12,7 & 77,4 \\
\hline & Trabalho em equipe dentro das unidades & 25,6 & 27 & 47,4 \\
\hline & Abertura da comunicação & 20,1 & 34,1 & 45,8 \\
\hline & Retorno da informação e comunicação sobre os erros & 19,9 & 30,0 & 50,1 \\
\hline & Resposta não punitiva aos erros & 50,5 & 20,3 & 29,2 \\
\hline & Adequação de pessoal & 37,7 & 20,3 & 42,0 \\
\hline \multirow{2}{*}{ Resultados } & Percepção geral de segurança do paciente & 25,7 & 14,3 & 60,0 \\
\hline & Frequência de eventos relatados & 12,6 & 14,2 & 73,2 \\
\hline
\end{tabular}


O menor tempo de atuação pode vir a ser um aspecto facilitador para moldar os profissionais na cultura organizacional, uma vez que eles são admitidos e apresentados aos princípios norteadores da instituição ${ }^{14}$. A instituição estudada tem implantada a metodologia de diferentes programas de acreditação, o que implica passar por processos de mudança intensos. Estudo revelou que os profissionais que trabalhavam em serviços acreditados se sentiam mais bem preparados para atender às necessidades dos usuários à medida que conseguiam trabalhar com recursos materiais, técnicos e humanos mais qualificados, padronização de processos e procedimentos e mais organização. Além disso, o clima organizacional era favorecido pelo processo de acreditação ${ }^{15}$.

O índice alfa de Cronbach mede a correlação entre as respostas do questionário e baseia-se na correlação média entre as perguntas. Portanto, é esperado encontrar variações entre as percepções dos indivíduos, por serem sujeitos sociais e com experiências diferentes. Os achados encontrados corroboram estudos nacionais (variabilidade de 0,52 a 0,91$)^{11}$ ou estrangeiros (variabilidade de 0,61 a 0,86$)^{16}$.

No que tange às dimensões sobre segurança, o estudo apresentou uma dimensão forte, sete neutras e quatro frágeis, o que sugere que muitas áreas devem ser aprimoradas. Além disso, algumas dimensões que estão no intervalo neutro estão próximas a $50 \%$ das respostas positivas, como "Trabalho em equipe entre as unidades", "Passagem de plantão/turno e transferência", "Expectativas do supervisor/chefe e ações promotoras de segurança" e "Retorno da informação e comunicação sobre os erros".

Em comparação a um estudo americano que avaliou a percepção dos profissionais que trabalham em CC, a distribuição das dimensões apresenta alguma similaridade, isto é, há apenas uma área forte, que é o "Trabalho em equipe dentro das unidades" (75,9\%), e como áreas fracas foram identificadas três dimensões: "Trabalho em equipe entre as unidades" (48,7\%), "Passagem de plantão/turno e transferência" (37,2\%) e "Resposta não punitiva aos erros" (37,3\%), sendo esta última também encontrada no presente estudo ${ }^{17}$.

Por tratar-se de hospital acreditado, pensou-se a princípio que as dimensões teriam respostas positivas em maior número, uma vez que as avaliações de qualidade impõem processos de melhoria contínuos e há a necessidade de apresentar resultados, estimulando os profissionais a se envolverem nos programas de qualidade. No entanto, ao analisar um estudo realizado no serviço de enfermagem de um hospital acreditado na Turquia, verificou-se que as dimensões da cultura não refletem, necessariamente, o processo de qualidade, apresentando como dimensões fortes: "Trabalho em equipe entre as unidades" (76\%) e "Percepção geral de segurança do paciente" (75\%); e duas dimensões frágeis: "Resposta não punitiva aos erros" (33\%) e "Adequação de pessoal" (22\%), também encontradas no presente estudo ${ }^{6}$.

Todavia, a gestão de uma instituição, seja globalmente, seja setorialmente, deve atentar para que os processos de melhoria sejam fundamentados em uma cultura positiva de segurança do paciente, independentemente do instrumento de avaliação a ser utilizado ${ }^{8}$. Os achados apontam para a necessidade de se olhar mais detalhadamente para quatro dimensões, visto tratar-se de setor tão importante e complexo.

A dimensão "Trabalho em equipe dentro das unidades" alerta para o fato de que, nesse setor, deve haver um clima de trabalho cordato, com relações interpessoais e multiprofissionais harmoniosas, com respeito às divergências de opiniões e sem comportamentos intimidadores, para que se possa trabalhar com tranquilidade e promover ações que garantam a segurança do paciente ${ }^{5}$. Diferentemente dos dados desta pesquisa, estudo realizado em CC com todos os profissionais que nele atuam indicou que "Clima de trabalho em equipe" era o que tinha maior escore, apontando para o papel do enfermeiro como um agente facilitador, com habilidades e potencialidades para a promoção desses valores ${ }^{8}$.

A dimensão "Abertura da comunicação" traz o entendimento de que os profissionais devem ter liberdade para se manifestar e apontar aspectos que possam colocar a segurança do paciente em risco. A literatura revela a dificuldade dos profissionais de enfermagem em se posicionar quando percebem algo errado, muitas vezes condicionada pela postura do profissional médico que entende esses alertas como críticas ao seu trabalho ${ }^{5}$. Portanto, ações que promovam a confiança dos profissionais para agir proativamente quando algo parece não estar dando certo devem ser estimuladas, a fim de proteger o paciente nesse cenário de assistência ${ }^{4}$.

A dimensão "Resposta não punitiva aos erros" também apresenta fragilidade, e estudos sobre cultura de segurança em CC sugerem que essa é uma das dimensões com menor número de respostas positivas, indicando preocupações para esse contexto dentro das instituições ${ }^{6,17}$. Estudos destacam que os profissionais de saúde se recusam a relatar erros por medo de serem penalizados e que a cultura da punição ainda é comum nos hospitais em todo o mundo e também no Brasil ${ }^{16}$. Sabe-se que a subnotificação de eventos adversos é uma questão que precisa ser trabalhada nos hospitais e em setores como o CC, para que se promova a segurança do paciente em ambientes não punitivos, estimulando os relatos 
de incidentes. Essas discrepâncias podem ser explicadas em função das diferenças no comportamento organizacional entre contextos culturais ou pela falta de políticas e procedimentos relacionados ao relato de erros ${ }^{6}$.

Por fim, os achados em relação à "Adequação de pessoal" convergem com os de estudos que identificam essa dimensão sistematicamente como frágil, seja na instituição de modo global, seja em setores específicos, como o examinado na presente pesquisa ${ }^{6,16,18}$.

O dimensionamento de pessoal é um tema crítico para os gestores da área de saúde, em função dos gastos com recursos humanos, como também o é para aqueles que atuam no cuidado direto ao paciente, com escala deficitária e maior risco de ocorrência de eventos adversos. Estudos confirmam essa informação, indicando que ainda hoje a escala de pessoal não é suficiente para as demandas de cuidado em muitas instituições de saúde, incluindo áreas críticas ${ }^{19}$.

Este estudo tem como limitação ter sido conduzido apenas com a equipe de enfermagem. Portanto, os resultados podem não refletir a cultura de segurança do paciente de outros profissionais de saúde que atuam no serviço. Também, são desconhecidos as intervenções de melhorias e os impactos na assistência que possam ter advindo da pesquisa em questão.

\section{CONCLUSÃO}

Os resultados sobre a percepção da cultura de segurança por profissionais de enfermagem em um CC revelaram fragilidades em quatro dimensões: "Trabalho em equipe dentro das unidades", "Abertura da comunicação", "Resposta não punitiva aos erros" e "Adequação de pessoal", com resultados inferiores a $50 \%$ das respostas positivas. Além disso, outras quatro dimensões, como "Trabalho em equipe entre as unidades", "Passagem de plantão/turno e transferência", "Expectativas do supervisor/chefe e ações promotoras de segurança" e "Retorno da informação e comunicação sobre os erros", apresentaram resultados que se encontram no intervalo neutro, porém mais próximos a $50 \%$. A dimensão "Aprendizado organizacional e melhoria contínua" foi caracterizada como a área forte da instituição $(77,4 \%)$.

Com isso, é necessário implementar mudanças que requeiram esforços de toda a organização hospitalar nos níveis estratégico, administrativo e operacional, principalmente para incentivar a atenção dos profissionais na condução das ações, de modo a fortalecer a cultura não punitiva, e estudar o dimensionamento de profissionais para o atendimento do paciente no perioperatório.

\section{REFERÊNCIAS}

1. Organização Mundial da Saúde. Divisão de Segurança do Doente, Departamento da Qualidade na Saúde. Estrutura conceitual da classificação internacional sobre segurança do doente. Relatório Técnico Final. Portugal: Organização Mundial da Saúde; 2011 [acessado em 10 out. 2018]. Disponível em: https://proqualis.net/sites/proqualis.net/files/ Estrutura\%20Conceitual\%20da\%20Classifica\%C3\%A7\%C3\%A30\%20 Int\%20Seguran\%C3\%A7a\%20do\%20Paciente.pdf

2. Lourenção DCA, Tronchin DM. Patient safety in the surgical environment: translation and cross-cultural adaptation of validated instrument. Acta Paul Enferm. 2016;29(1):1-8. http://dx.doi. org/10.1590/1982-0194201600002

3. Carvalho PA, Göttems LBD, Pires MRGM, Oliveira MLCl. Safety culture in the operating room of a public hospital in the perception of health care professionals. Rev Latino-Am Enferm. 2015;23(6):1041-8. http:// dx.doi.org/10.1590/0104-1169.0669.2647

4. World Health Organization. WHO Guidelines for safe surgery 2009: safe surgery saves lives: the second global patient safety challenge [Internet]. Genebra: WRO; 2009 [acessado em 20 out. 2018]. Disponível em: http:// apps.who.int/iris/bitstream/handle/10665/44185/9789241598552_eng. pdf;jsessionid=72CC6373244DEA74629F67F2933B9D72? sequence=1
5. Halverson AL, Neumayer L, Dagi TF. Leadership skills in the OR: Part II: Recognizing disruptive behavior. Bull Am Coll Surg. 2012;97(6):17-23.

6. Gözlü K, Kaya S. Patient safety culture asp by nurses in a Joint Commission International Accredited Hospital in Turkey and its comparison with Agency for Healthcare Research and Quality Data. Patient Saf Qual Improv [Internet]. 2016 [acessado em 20 out. 2018];4(4):441-9. Disponível em: http://psj.mums.ac.ir/article_7640. html. http://dx.doi.org/10.22038/PSJ.2016.7640

7. Halligan M, Zecevic A. Safety culture in healthcare: a review of concepts, dimensions, measures and progress. BMJ Qual Safety. 2011;20(4):338-43. http://dx.doi.org/10.1136/bmjqs.2010.040964

8. Correggio TC, Amante LN, Barbosa SFF. Avaliação da cultura de segurança do paciente em centro cirúrgico. Rev SOBECC. 2014;19(2):6773. http://dx.doi.org/10.4322/sobecc.2014.012

9. Brasil. Portaria n. 529, de $1^{\circ}$ de abril de 2013. Institui o Programa Nacional de Segurança do Paciente (PNSP) [Internet]. Brasília: Ministério da Saúde; 2013 [acessado em 22 out. 2018]. Disponível em: http://www.saude.mt.gov.br/upload/controle-infeccoes/pasta2/ portaria-msgm-n-529-de-01-04-2013.pdf 
10. Sorra JS, Nieva VF. Hospital survey on patient safety culture (Prepared by Westat, under Contract No. 290-96-0004). AHRQ Publication No. 04-0041. Rockville; 2004.

11. Reis CT, Laguardia J, Vasconcelos AGG, Martins M. Reliability and validity of the Brazilian version of the Hospital Survey on Patient Safety Culture (HSOPSC): a pilot study. Cad Saúde Pública. 2016;32(11):e00115614. http://dx.doi.org/10.1590/0102-311x00115614

12. Fiocruz. Pesquisa inédita traça perfil da enfermagem no Brasil. Agência Fiocruz de Notícias [Internet]. 2015 [acessado em 22 out. 2018] Disponível em: https://portal.fiocruz.br/pt-br/content/ pesquisa-inedita-traca-perfil-da-enfermagem-no-brasil

13. Günes ÜY, Gürlek Ö, Sönmez M. A survey of the patient safety culture of hospital nurses in Turkey. Collegian. 2016;23(2):225-32. http:// dx.doi.org/10.1016/j.colegn.2015.02.005

14. Paula P, Stefano SR, Andrade SM, Zampier MA. Clima e cultura organizacional em uma organização. Gestão Regionalidade [Internet]. 2011 [acessado em 20 out. 2018];27(81). Disponível em: https://doi. org/10.13037/gr.vol27n81.1279

15. Manzo BF, Ribeiro HCTC, Brito MJM, Alves M. A enfermagem no processo de acreditação hospitalar: atuação e implicações no cotidiano de trabalho. Rev Latino-Am Enferm. 2012;20(1):151-8. http://dx.doi. org/10.1590/S0104-11692012000100020
16. Najjar S, Baillien E, Vanhaecht K, Hamdan M, Euwema M, Vleugels $A$, et al. Similarities and differences in the associations between patient safety culture dimensions and self-reported outcomes in two different cultural settings: a national cross-sectional study in Palestinian and Belgian hospitals. BMJ Open [Internet]. 2018 [acessado em 22 out. 2018];8:e021504. Disponível em: https:// bmjopen.bmj.com/content/8/7/e021504. http://dx.doi.org/10.1136/ bmjopen-2018-021504

17. Adams-Pizarro I, Walker Z, Robinson J, Kelly S, Toth M. Using the AHRQ Hospital Survey on Patient Safety Culture as an intervention tool for regional clinical improvement collaboratives [Internet]. Rockville: Agency for Healthcare Research and Quality (US); 2008 [acessado em 22 out. 2018]. v. 2. Disponível em: https://www.ncbi. nlm.nih.gov/books/NBK43728/

18. Silva-Batalha EMS, Melleiro MM. Cultura de segurança do paciente em um hospital de ensino: diferenças de percepção existentes nos diferentes cenários dessa instituição. Texto Contexto Enferm. 2015;24(2):432-41. http://dx.doi. org/10.1590/0104-07072015000192014

19. Gonçalves LA, Andolhe R, Oliveira EM, Barbosa RL, Faro ACM, Gallotti RMD, et al. Nursing allocation and adverse events/incidents in intensive care units. Rev Esc Enferm USP. 2012;46(Núm. Spe.):71-7. http://dx.doi.org/10.1590/ S0080-62342012000700011 